\title{
Erratum to: Generalized individual dental age stages for fossil and extant placental mammals
}

\section{Ulrike Anders • Wighart von Koenigswald •}

\author{
Irina Ruf $\cdot$ B. Holly Smith
}

Published online: 31 March 2011

(C) Springer-Verlag 2011

\section{Erratum to: Paläontol Z}

\section{DOI 10.1007/s12542-011-0098-9}

During processing of this article, inappropriate changes were made to Table 1 that rendered its content incomprehensible. The correct version of the table is given here.

Table 1 Correlation of the IDAS stages to age determinations of different species to illustrate the transferability of the IDAS system

\begin{tabular}{|c|c|c|c|c|c|c|}
\hline \multirow[t]{2}{*}{ IDAS } & \multirow{2}{*}{$\begin{array}{l}\text { Common } \\
\text { use } \\
\text { but rarely } \\
\text { defined }\end{array}$} & \multicolumn{2}{|c|}{$\begin{array}{c}\text { Schultz } \\
\text { (primates) }\end{array}$} & \multirow{2}{*}{$\begin{array}{c}\text { Knußmann } \\
1988 \\
\text { (humans) }\end{array}$} & \multirow[t]{2}{*}{$\begin{array}{l}\text { Grau } 1970 \\
\text { (racoons) }\end{array}$} & \multirow[t]{2}{*}{$\begin{array}{c}\text { Goddard } 1970 \\
\text { (rhinoceros) }\end{array}$} \\
\hline & & 1935 & 1960 & & & \\
\hline 0 & prenatal & & & fetus & & \\
\hline 1 & - & infantile & & infans I, II & & class I-VI \\
\hline 2 & juvenine & juvenile & syoung & juvenis & & class VII-XI \\
\hline 3 & adult & & & adultus/ & class I-III & class XII-XVI \\
\hline 4 & tate & adult & adult & matures & class IV & class XVII-XVIII \\
\hline 5 & senile & & old & senilis & class $\mathrm{V}$ & class XIX-XX \\
\hline
\end{tabular}

The online version of the original article can be found under doi:10.1007/s12542-011-0098-9.

U. Anders $(\varangle)$. W. von Koenigswald · I. Ruf

Steinmann-Institut für Geologie, Mineralogie und Paläontologie,

Rheinische Friedrich-Wilhelms-Universität Bonn, Nussallee 8,

53115 Bonn, Germany

e-mail: uanders@uni-bonn.de

B. H. Smith

Museum of Anthropology, The University of Michigan,

Ann Arbor, MI 48109-1107, USA 